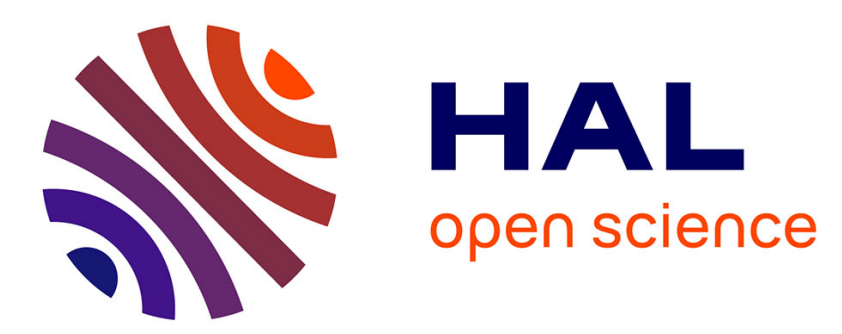

\title{
Perfectionnements apportés à la mesure de la quantité d'ions collectée par un spectrographe de masse à étincelles
}

\author{
F. Degrève, C. Le Bars, R. Dablainville, R. Figaret
}

\section{To cite this version:}

F. Degrève, C. Le Bars, R. Dablainville, R. Figaret. Perfectionnements apportés à la mesure de la quantité d'ions collectée par un spectrographe de masse à étincelles. Revue de Physique Appliquée, 1970, 5 (4), pp.627-631. 10.1051/rphysap:0197000504062700 . jpa-00243436

\section{HAL Id: jpa-00243436 https://hal.science/jpa-00243436}

Submitted on 1 Jan 1970

HAL is a multi-disciplinary open access archive for the deposit and dissemination of scientific research documents, whether they are published or not. The documents may come from teaching and research institutions in France or abroad, or from public or private research centers.
L'archive ouverte pluridisciplinaire HAL, est destinée au dépôt et à la diffusion de documents scientifiques de niveau recherche, publiés ou non, émanant des établissements d'enseignement et de recherche français ou étrangers, des laboratoires publics ou privés. 


\title{
PERFECTIONNEMENTS APPORTÉS A LA MESURE DE LA QUANTITÉ D'IONS COLLECTÉE PAR UN SPECTROGRAPHE DE MASSE A ÉTINCELLES
}

\author{
F. DEGRÈVE, C. LE BARS, R. DABLAINVILLE et R. FIGARET \\ Centre de Recherches du Groupe Péchiney, B. P. 24, 38, Voreppe, France
}

(Reçu le 25 février 1970)

\begin{abstract}
Résumé. - Deux modifications technologiques ont été opérées sur un spectrographe de masse à étincelles destiné à l'analyse des solides.

Il s'agit d'une part de la construction d'un nouvel ensemble amplificateur-intégrateur (moniteur) de hautes performances destiné à mesurer le nombre de charges collecté et d'autre part de l'installation d'un atténuateur de faisceau ionique.

Les premiers résultats obtenus à la suite de l'utilisation simultanée de ces deux dispositifs montrent qu'il est possible de doser de façon significative, quoique relativement imprécise le constituant principal d'un échantillon et avec précision des impuretés à teneur moyenne.

Abstract. - Two technological modifications have been made on a spark source mass spectrograph used for solid analysis : firstly, the construction of a new amplifier-integrator device intended to measure the quantity of collected charges and secondly the installation of an ion beam chopper.

The first results obtained, after the coupling of the two devices, have shown that it is possible to analyze significantly, but with a relative imprecision, the principal constituant (matrix) and, with precision, impurities.
\end{abstract}

Introduction. - En spectrographie de masse à étincelles, le dosage d'un constituant au sein d'un échantillon solide requiert la mesure de deux variables physiques [1] : le noircissement de raies non saturées détectées par la plaque photographique et le nombre total d'ions collecté pour un temps de pause donné. $\mathrm{Si}$ le faisceau ionique est représentatif du solide soumis à l'étincelle, cette charge totale, ou encore exposition totale, est proportionnelle à la quantité d'ions monochargés relative à chaque constituant. La variation $\mathrm{du}$ noircissement des raies en fonction de l'exposition totale représente la courbe de réponse de la plaque. Dans la portion linéaire de cette courbe, il est possible de définir une exposition $E_{50}$ caractéristique d'une raie de noircissement donné, par exemple de transmission $T=50 \%$. Au rendement d'ionisation près, $E_{50}$ est alors inversement proportionnelle à la teneur $C \mathrm{du}$ constituant dans le solide :

$$
E_{50} \div 1 / C
$$

ou encore

$$
K \div C
$$

en posant

$$
E_{50}=1 / K
$$

Ainsi, le dosage d'un constituant à forte teneur tel que la matrice, comporte une série d'expositions totales de faible valeur caractérisées par des temps de pause de l'ordre de la fraction de seconde. La mesure de celles-ci à l'aide d'un collecteur intermédiaire (moniteur) disposé sur la trajectoire du faisceau ionique avant que ne s'opère la sélection selon le rapport masse/charge est rendue aléatoire, sinon impossible, en raison des instabilités et dérives affectant l'amplificateur de mesure.

Pour pallier ces inconvénients, deux modifications technologiques ont été opérées sur un spectrographe MS 7 (A. E. I.) :

- la construction d'un nouveau moniteur de performances supérieures,

- l'installation d'un atténuateur de faisceau ionique permettant d'accroître le temps de pause des expositions, c'est-à-dire la durée de leur mesure.

Aux expositions moyennes (teneurs moyennes), où le problème de la stabilité se pose de façon moins critique, l'atténuateur offre la possibilité de maintenir constants les paramètres d'étincelle tout en accroissant la consommation d'échantillon.

Les performances analytiques obtenues à la suite de l'utilisation simultanée de ces deux dispositifs ont été étudiées aux faibles et moyennes expositions de façon à préciser les conditions opératoires donnant lieu à une dispersion minimale des résultats. 
1. Aspect expérimental. - 1.1 Dispositif DE MESURE DU NOMBRE D'IONS. - Le courant ionique instantané est mesuré par l'intermédiaire d'une différence de potentiel créée aux bornes d'une résistance de $10^{10} \Omega$. Après amplification, son intégration au cours du temps permet de déduire le nombre total de charges transmis au détecteur photographique. L'ensemble amplificateur-intégrateur réalisé utilise des composants électroniques modernes. Il se différencie du modèle antérieur par deux modifications importantes :

- meilleure stabilité de l'amplificateur pour une même sensibilité,

- reproductibilité accrue par l'automatisation des mesures au niveau du circuit intégrateur.

1.1.1 Amplificateur de mesure. - L'étage d'entrée est constitué par un tube électromètre double, suivi d'un amplificateur opérationnel en montage symétrique, un fort taux de contre-réaction stabilisant l'ensemble. Les différents gains en tension $(100,30,10,3$ et 1$)$ sont obtenus à l'aide d'un commutateur à la disposition de l'opérateur. Ces éléments, soigneusement découplés et protégés des inductions, sont disposés à proximité immédiate de la cible collectrice d'ions; ils constituent la « tête de mesure ». Les signaux résultants sont transmis sous faible impédance au boîtier de contrôle et appliqués à un deuxième amplificateur opérationnel fournissant un gain supplémentaire de valeurs 1, 3 ou 10 (voir Fig. 1).

Ce dernier élément délivre l'information "mesure du courant ionique » à un galvanomètre à lecture directe d'une part et au circuit intégrateur d'autre part.
Le réglage de zéro qui s'effectue par variation de la tension écran du tube électromètre compensateur est ainsi pratiquement indépendant de la gamme de mesure choisie, ce qui évite de fréquentes retouches. L'ensemble est alimenté en tension continue fortement stabilisée par deux sources de +15 et -15 volts. Leur coefficient de régulation est de l'ordre de $5 \times 10^{-6}$ (réseau et charge) et leur coefficient de température voisin de $1 \times 10^{-5} /{ }^{\circ} \mathrm{C}$.

Dans sa nouvelle version, l'amplificateur permet, pour une même stabilité, de mesurer des valeurs 10 fois plus faibles du courant ionique instantané et donc d'enregistrer des expositions dix fois plus petites.

1.1.2 Circuit intégrateur; automatisation de la mesure. - Le circuit intégrateur qui reçoit directement le signal de sortie de l'étage précédent comporte un amplificateur opérationnel à faible dérive et très grande impédance d'entrée, utilisant dans la boucle de contreréaction une capacité de précision au mylar (voir Fig. 2).

Le gain de l'étage intégrateur peut être ajusté aux valeurs $100,30,10,3$ ou 1 à l'aide d'un commutateur. Le signal de sortie est dirigé d'une part vers un galvanomètre à lecture directe et d'autre part, par l'intermédiaire d'un étage séparateur (opérationnel, non inverseur, au gain unité) vers un étage comparateur. Ce dernier reçoit sur sa deuxième entrée une tension stable, mais réglable par un potentiomètre linéaire à 10 tours, entre 0 et 10 volts, soit entre 0 et $100 \%$ de la pleine échelle de l'intégrateur. Lors de la coïncidence entre la tension issue de l'intégrateur et celle réglée

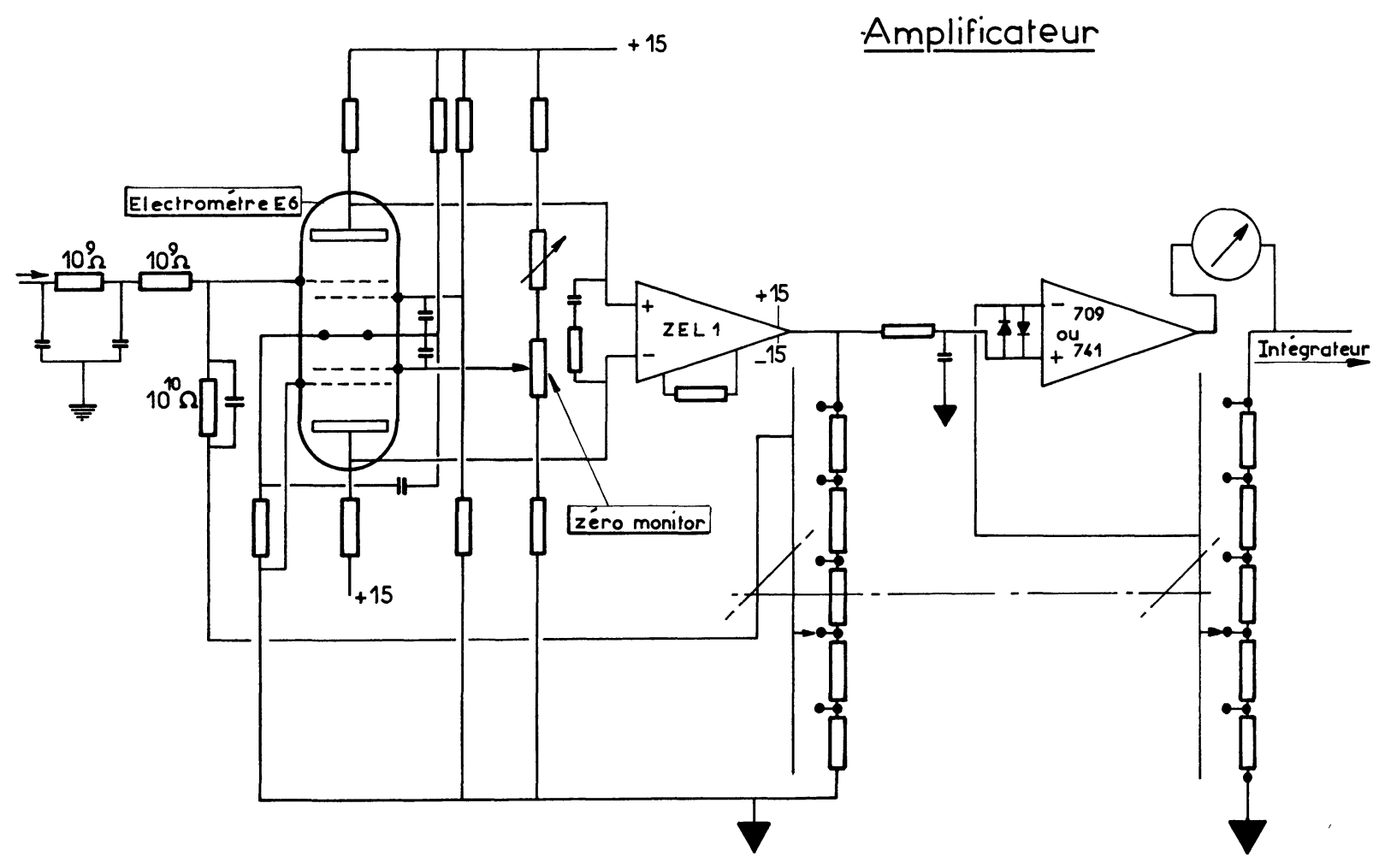

FIg. 1. - Schéma de l'amplificateur. 


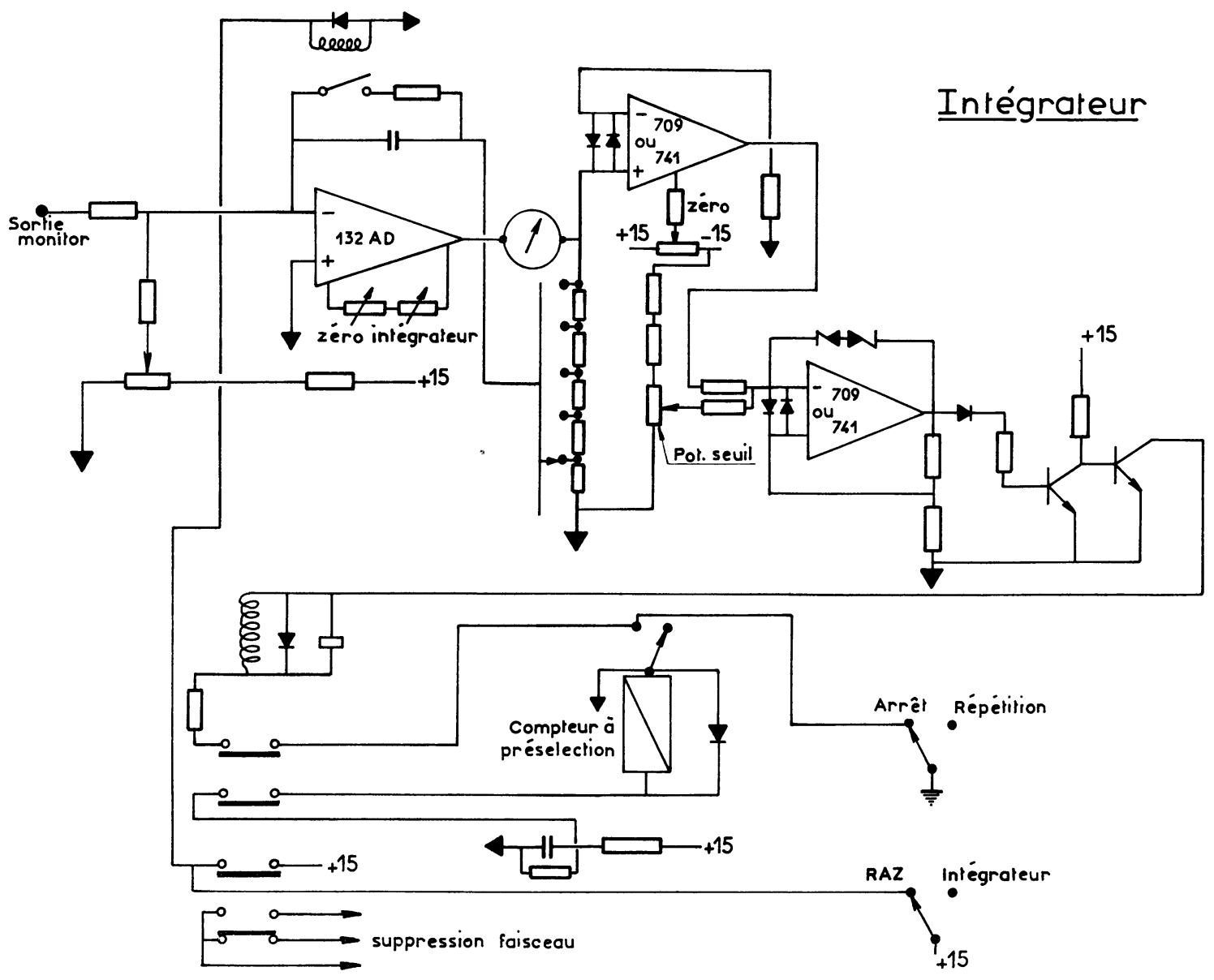

Fig. 2. - Schéma du circuit intégrateur et d'automatisme des mesures.

en référence, le comparateur actionne un relais qui effectue les opérations suivantes : remise à zéro de l'intégrateur, suppression du faisceau d'ions et avance d'un pas sur un compteur d'impulsions électromagnétique muni d'une prédétermination. Il est ainsi possible de définir à l'avance le nombre d'échelles d'intégration, le cycle étant reproductible sur toutes les gammes de mesure. Un inverseur accessible sur le panneau de contrôle permet de choisir le mode de mesure : soit intégration unique, soit plusieurs intégrations successives dont le nombre est prédéterminé par le compteur électromagnétique.

1.2 Atténuateur de faisceau ionique. - Ce dispositif, décrit de façon plus détaillée ailleurs [2-4] a pour fonction d'interrompre périodiquement la transmission des ions vers la plaque, en polarisant une électrode de l'optique ionique par un signal répulsif d'amplitude et longueur d'impulsion constantes, mais de fréquence $f$ réglable selon l'exposition choisie. Pour une exposition donnée, l'accroissement du temps de pause résultant est donné par :

$$
r=t_{a} / t=1 /\left(f \cdot t_{i}\right)
$$

où $t_{a}, t$ et $t_{i}$ sont respectivement les temps de pause avec atténuation et sans atténuation et le temps de passage du faisceau lors d'une impulsion. Ceci permet d'aug- menter la consommation d'échantillon tout en maintenant constants les paramètres d'étincelle (longueur d'impulsion $1(\mu \mathrm{s})$ et fréquence de répétition $F(\mathrm{~Hz})$ ). L'importance relative de l'accroissement du temps de pause et de la consommation d'échantillon sera discutée plus loin.

2. Résultats analytiques. -2.1 MeSURE DES PARAMÈTRES ANALYTIQUES. - Afin de mesurer significativement une amélioration éventuelle apportée par l'une des modifications technologiques décrites plus haut, les données brutes d'analyse (exposition, noircissement et largeur des raies, noircissement du fond continu adjacent) sont traitées par ordinateur [5] de façon à décrire mathématiquement la courbe de réponse correspondante par la relation semi-empirique de Hull [6-7]. Celle-ci est fonction de 2 paramètres :

- $R$, constante proportionnelle au contraste de la plaque photographique, et

- $K$ défini par la relation (3).

Le programme [5] permet en outre de connaître l'écart type relatif $\sigma(K)$ affectant la valeur de $K$ et la teneur $C_{i}$ d'une impureté $i$ à partir d'un élément de référence de teneur $C_{\boldsymbol{r}}$. La relation (2) s'exprime alors sous la forme :

$$
C_{i}=C_{r} \cdot\left(K_{i} / K_{r}\right) \cdot\left(M_{i} / M_{r}\right) \cdot S_{i} \quad \text { (ppm poids) }
$$


où $M$ est la masse molaire et $S_{i}$ le coefficient de sensibilité de $i$ relativement à $r$.

2.2 Courbe DE RÉPONSE DE $\mathrm{CU}^{+}$MATRICE. - Il est toujours intéressant de pouvoir disposer du constituant principal comme élément de référence. La stabilité de l'amplificateur du moniteur conventionnel aux expositions les plus faibles ne permettrait pas la mesure suffisamment précise de couples expositions-raies non saturées indispensables à la construction de la courbe de réponse matrice. Seuls des éléments polyisotopiques comportant un isotope de faible abondance $(\leqslant 1 \%)$ pouvaient être utilisés comme étalon interne.

L'exemple suivant montre les résultats obtenus grâce au nouveau moniteur pour un échantillon de cuivre pur, c'est-à-dire pour un élément dont l'abondance des deux isotopes 63 et 65 est relativement élevée, $69,1 \%$ et $30,9 \%$ respectivement.

Deux plaques comportant chacune 16 expositions comprises entre $10^{-4}$ et $10^{-2}$ nanocoulombs ( $\mathrm{nClb}$ ) ont été réalisées en gardant constants les paramètres d'étincelle $l$ et $F$. L'une d'elles, correspondant à un faisceau non atténué, a permis d'établir une courbe de réponse de ${ }^{63} \mathrm{Cu}^{+}$et ${ }^{65} \mathrm{Cu}^{+}$à partir de 27 raies non saturées, l'autre, correspondant à un faisceau atténué, comporte 16 raies exploitables. Le tableau I résume les conditions d'expérience ainsi que les valeurs obtenues pour les paramètres caractéristiques de la courbe de réponse : $K, \sigma(K)$ et $R$. Pour les deux courbes établies, la valeur de $R$ est correcte; elle est en effet généralement comprise entre 1,0 et 1,5 pour les plaques Ilford Q2 utilisées dans ce travail [7]. Par contre, la valeur de $\sigma(K)$ est abaissée d'un facteur 5 lorsque le faisceau est atténué. Ceci doit être attribué au fait que le moniteur, caractérisé d'une part par une meilleure reproductibilité dans la définition d'une exposition et d'autre part par un temps de réponse de l'ordre de $10^{-3} \mathrm{~s}$, peut effectuer la mesure pendant une période de
TABLEAU I

Paramètres caractéristiques de la courbe de réponse de $\mathrm{Cu}^{+}$matrice avec et sans atténuation de faisceau

\begin{tabular}{|c|c|c|}
\hline Echantillon & \multicolumn{2}{|c|}{ Matrice $(C=100 \%)$} \\
\hline Conditions & $\begin{array}{l}\text { sans atténua- } \\
\text { tion }\end{array}$ & $\begin{array}{l}\text { avec atténua- } \\
\text { tion } \\
f=0,2 \mathrm{kHz} \\
t_{i}=5 \mu \mathrm{s}\end{array}$ \\
\hline Paramètres & $F=10 \mathrm{~Hz}$ & $F=300 \mathrm{~Hz}$ \\
\hline Etincelle & $l=25 \mu \mathrm{s}$ & $l=200 \mu \mathrm{s}$ \\
\hline Isotope & $\sigma(K) \%$ & $\sigma(K) \%$ \\
\hline${ }^{63} \mathrm{Cu}^{+}$ & 89,4 & 19,5 \\
\hline${ }^{65} \mathrm{Cu}^{+}$ & 70,8 & 20,8 \\
\hline$\sigma(K)$ total $\%(*)$ & 114,9 & 21,8 \\
\hline$R$ & 1,29 & 1,01 \\
\hline$K(* *)$ & $7,16 \times 10^{2}$ & $2,46 \times 10^{3}$ \\
\hline
\end{tabular}

$\left(^{*}\right)$ Les paramètres $R$ et $K$ sont calculés pour l'ensemble des isotopes, $\sigma(K)$ représente la dispersion caractérisant la courbe de réponse établie à l'aide d'un seul isotope et $\sigma(K)$ total, celle affectant une courbe établie pour l'ensemble des isotopes [5].

(**) Les écarts observés sur les valeurs de $K$ sont dus essentiellement aux différences de sensibilité des plaques.

temps plus longue. L'accroissement $r$ défini par l'équation (4) est, dans ces conditions particulières, égal à $10^{3}$, ce qui amène le temps de mesure à environ $1 \mathrm{~s}$. La consommation d'échantillon qui est augmentée dans les mêmes proportions ne peut entrer en ligne de compte dans le gain de reproductibilité des résultats puisqu'il s'agit du constituant principal qui est supposé réparti de façon homogène au sein de l'échantillon. Même lorsque le faisceau d'ions matrice est atténué, la valeur de $\sigma(K)$ peut paraître élevée, en tout cas plus élevée que celle observée pour des ions impureté (voir 2.3 et tableau II). Il faut alors tenir compte de deux causes

\section{TABleaU II}

Analyse quantitative d'un échantillon d'aluminium - Teneur C en ppm poids

\begin{tabular}{|c|c|c|c|c|c|c|c|c|c|c|c|}
\hline \multirow[b]{2}{*}{ Impureté } & \multirow[b]{2}{*}{$\begin{array}{l}\text { Teneur } \\
\text { annoncée }\end{array}$} & \multirow[b]{2}{*}{$S_{i}$} & \multicolumn{3}{|c|}{ Ancien moniteur } & \multicolumn{3}{|c|}{ Nouveau moniteur } & \multicolumn{3}{|c|}{$\begin{array}{l}\text { Nouveau moniteur } \\
\text { atténuateur }(* *)\end{array}$} \\
\hline & & & $C$ & $\begin{array}{c}\sigma(K) \\
\%\end{array}$ & $R$ & $C$ & $\begin{array}{c}\sigma(K) \\
\%\end{array}$ & $R$ & $C$ & $\begin{array}{c}\sigma(K) \\
\%\end{array}$ & $R$ \\
\hline - & - & - & - & - & - & - & - & - & - & - & - \\
\hline $\mathrm{Cu}(*)$ & 580 & 1,00 & 580 & 5,2 & 1,05 & 580 & 17,5 & 1,09 & 580 & 9,1 & 1,08 \\
\hline $\mathrm{Cr}$ & 110 & 0,71 & 74 & 29,6 & 1,59 & 152 & 10,5 & 1,01 & 121 & 8,0 & 1,03 \\
\hline $\mathrm{Mn}$ & 610 & 0,60 & 400 & 24,0 & 1,92 & 1000 & 17,8 & 1,05 & 548 & 8,2 & 1,03 \\
\hline $\mathrm{Fe}$ & 2400 & 0,95 & 2170 & 0,4 & 1,11 & 2730 & 13,1 & 1,09 & 2645 & 3,5 & 1,10 \\
\hline $\mathrm{Ni}$ & 95 & 1,05 & 74 & 19,3 & 1,25 & 84 & 43,3 & 1,12 & 99 & 10,3 & 1,09 \\
\hline Co & 2 & 0,94 & 2,5 & 6,2 & 1,11 & 2,1 & 16,5 & 1,00 & 2,1 & 4,8 & 1,02 \\
\hline $\mathrm{Zn}$ & 950 & 0,41 & 1165 & 17,3 & 1,20 & 2890 & 14,3 & 1,07 & 982 & 8,6 & 1,10 \\
\hline Ga & 90 & 0,60 & 84 & 22,5 & 1,24 & 134 & 29,5 & 1,10 & 100 & 10,3 & 1,08 \\
\hline$\overline{\sigma(\mathrm{K})}$ & & & & $21,8 \%$ & & & $20,3 \%$ & & & $7,8 \%$ & \\
\hline
\end{tabular}

(*) Etalon interne.

(**) $f=20 \mathrm{kHz}$. $t_{i}=5 \mu \mathrm{s}$. 
susceptibles de perturber la détection d'ions matrice : d'une part, les phénomènes de charges d'espace [8] qui déforment le profil des raies [9] et rendent moins précise leur exploitation quantitative, d'autre part des fluctuations aléatoires du taux d'ions polychargés [8] qui peuvent modifier la relation fonctionnelle existant entre la quantité d'ions monochargés et leur noircissement (courbe de réponse). Il est bien connu que ces effets perturbateurs sont moins prononcés pour des ions impureté.

De nombreux essais réalisés sur des échantillons d'aluminium, élément monoisotopique, ont montré que la stabilité du nouveau moniteur n'est pas encore suffisante pour établir complètement et de façon précise la courbe de réponse matrice. L'utilisation de celle-ci comme étalon interne n'est valable que pour des dosages semi-quantitatifs d'impuretés.

2.3 COURBE DE RÉPONSE D'IMPURETÉS ANALYSE QUANTITATIVE. - Pour illustrer aux teneurs moyennes les performances du nouveau moniteur et de son couplage à l'atténuateur, le dosage quantitatif de 7 impuretés métalliques contenues dans un échantillon témoin d'aluminium a été réalisé selon trois modes de détection utilisant respectivement l'ancien moniteur, le nouveau moniteur et le couple nouveau moniteuratténuateur. Le cuivre, présent à $580 \mathrm{ppm}$ poids a été choisi comme étalon interne. Pour chaque impureté, le coefficient $S_{\imath}$ intervenant dans la relation (5) a été au préalable déterminé à l'aide d'une série d'échantillons étalons d'aluminium.

Les résultats figurent dans le tableau II.

Dans ce domaine de teneurs correspondant à des expositions supérieures à $0,1 \mathrm{nClb}$, le nouveau moniteur rend plus homogène le profil des différentes courbes (les différents $R$ se situent dans une fourchette de valeurs plus restreinte) mais la stabilité de l'amplificateur qui joue un rôle moins critique dans cette gamme d'expositions n'engendre pas un gain de pré- cision significatif. Ce dernier, s'il existe, est sûrement masqué par une imprécision globale liée à d'autres facteurs tels que l'hétérogénéité des échantillons à l'échelle du volume consommé et la modification des paramètres d'étincelle $l$ et $F$.

C'est en exerçant son influence sur ces deux points essentiels que l'atténuateur permet d'abaisser la dispersion moyenne $\bar{\sigma}$ de 21 à $8 \%$. Pour la fréquence d'atténuation imposée lors de cette analyse $(f=20 \mathrm{kHz})$ l'accroissement de consommation $r$ est égal à 10 , ce qui minimise les effets d'une répartition éventuellement hétérogène des impuretés tout en maintenant constants les paramètres d'étincelle.

3. Conclusion. - Les deux modifications technologiques décrites dans ce travail améliorent notablement les performances analytiques mais de façon distincte selon la zone des teneurs envisagées.

Le nouveau moniteur qui se différencie du modèle conventionnel par une meilleure reproductibilité et une stabilité 10 fois meilleure, permet, grâce à une atténuation de faisceau, d'accroître notablement le temps de pause des très faibles expositions et d'établir significativement la courbe de réponse de matrices polyisotopiques, ceci est toujours utile en analyse semi-quantitative.

Aux expositions moyennes caractérisées par un temps de pause suffisamment long, la stabilité de l'amplificateur joue un rôle moins important. Par contre, l'atténuateur améliore la précision du dosage quantitatif d'impuretés à teneur moyenne en accroissant la consommation d'échantillon et en maintenant constants les paramètres d'étincelle.

Remerciements. - Les auteurs tiennent à remercier M. Bril, Chef de la Section des Etudes Analytiques pour les encouragements qu'il nous a apportés au cours de la réalisation de ce travail.

\section{Bibliographie}

[1] Owens (E. B.) dans « Mass Spectrographic Analysis of Solids ), A. J. Ahearn (Editor), Elsevier, Amsterdam, 1966, p. 56.

[2] JACKSON (P. F. S.), WhiteheAd (J.) et Vossen (P. G. T.), Anal. Chem., 1967, 39, 1737.

[3] Vossen (P. G. T.), Anal. Chem., 1968, 40, 632.

[4] Brown (R.), Vossen (P. G. T.), Jackson (P. F. S.), et Whitehead (J.), "Advances in Mass Spectrometry ), vol. 4, p. 431, Ed. E. Kendrick, London, Institute of Petroleum, 1968.
[5] Degrève (F.) et Champetier de Ribes (D.), Int. J. Mass Spertrom. Ion Physics, 1970, 4, 125.

[6] Hull (C. W.), 10th Ann. Conf. Mass Spectrometry and Allied Topics, New Orleans, La., 1962.

[7] Woolston (J. R.), 14th Ann. Conf. Mass Spectrometry and Allied Topics, St Louis, Miss., 1965.

[8] Franzen (J.), Thèse de Doctorat, Mainz, 1963.

[9] Valeriano (P.), Cavard (A.), Stefani (R.), BourguilLOT (R.) et Desjardins (M.), Méthodes Physiques d'Analyse (GAMS), juillet-septembre 1966, p. 266. 